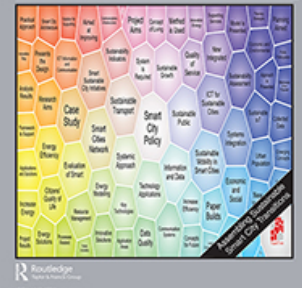

Journal of

ISSN: (Print) (Online) Journal homepage: https://www.tandfonline.com/loi/cjut20

\title{
Building City Dashboards for Different Types of Users
}

\section{Gareth W. Young, Rob Kitchin \& Jeneen Naji}

To cite this article: Gareth W. Young, Rob Kitchin \& Jeneen Naji (2021) Building City Dashboards for Different Types of Users, Journal of Urban Technology, 28:1-2, 289-309, DOI: 10.1080/10630732.2020.1759994

To link to this article: https://doi.org/10.1080/10630732.2020.1759994

\section{Published online: 02 Jun 2020.}

Submit your article to this journal

Џ Article views: 765

Q View related articles $\llbracket$

View Crossmark data $ऍ$

4

Citing articles: 4 View citing articles 


\title{
Building City Dashboards for Different Types of Users
}

\author{
Gareth W. Young (1D ${ }^{a}$, Rob Kitchin (1) ${ }^{b}$, and Jeneen Naji (D) ${ }^{c}$ \\ ${ }^{a}$ National Centre for Geocomputation, Maynooth University, Kildare, Ireland; ${ }^{b}$ Maynooth University Social \\ Sciences Institute and Department of Geography, Maynooth University, Kildare, Ireland; 'Department of \\ Media Studies, Maynooth University, Kildare, Ireland
}

\begin{abstract}
City dashboard websites are a common modality for bringing opengovernment philosophies into the public domain. Yet, there has been little research concerning the optimum design for city dashboards that takes account of users' expectations and skills. Indeed, there has been minimal exploration of user-centered design (UCD) to improve the usability and utility of smart city technologies in general. This study sought to conduct a user evaluation analysis to inform a UCD approach to city dashboards. Interviews with different types of users were conducted that applied a protocol analysis to gain insight into user perspectives and experiences of city dashboards. Along with critical incident technique procedures, interaction data of critical significance to the user was collected and a content analysis was conducted. These qualitative data were used to determine representations of users, as identified through observed behaviors, attitudes, needs, and goals. Targeted-scope user experience personas for the design process were then constructed to represent and build empathy towards three potential users of city dashboard systems: novices, end-users, and advanced users. The collected user requirements and the personas formulated are underpinning the re-design of an existing city dashboard.
\end{abstract}

\section{KEYWORDS}

City dashboards; smart cities; user requirements; user models; user-centered design

\section{Introduction}

Many city administrations seek to make local government data available to the public via open data portals (Lauriault and Francoli, 2017). Some also present a selection of these data via dashboards using data visualization tools (Kitchin et al., 2015). These dashboards often follow an open-government philosophy of transparency, designed to instill a sense of accountability for public institutions to citizens (Lněnička and Máchová, 2015; Lourenço, 2015; Geiger and Von Lucke, 2012). They can also be used internally within city administrations to track key performance indicators concerning operations and to manage city service provision (Behn, 2014). Generally, they are an important, public-facing element of a wider smart city approach to urban management and government (Kitchin and McArdle, 2017). Dashboard visualizations communicate data immediately relevant to citizens, while the temporal aspect enables a time-series analysis of the wider city 
(Hemmersam et al., 2015). They also facilitate community participation in planning and in shaping, implementing, and evaluating policy by empowering the public to undertake their own analysis and to participate in consultation (Bourgeois and Horan, 2007; Desouza and Bhagwatwar, 2014; Shin and Shin, 2012).

A city dashboard is commonly presented as a web-based user interface that organizes and presents city-specific data to a broad constituency of users, such as analysts, policymakers, politicians, and civil society. However, our contention, based on established findings in the data visualization and Human-Computer Interaction (HCI) literature, and informal user feedback concerning the initial version of the Dublin Dashboard, is that many city dashboards are too specialized and complex, requiring higher-level data literacy, to be easily used by the general public (Kitchin and McArdle 2017; Young and Kitchin, 2020). Moreover, previous studies have indicated that some improvements are required to enhance the openness and accessibility of open-data portals (Máchová and Lněnička, 2017; Attard et al., 2015; Zuiderwijk et al., 2014). In other words, city dashboards do not, at present, provide the transparency to citizens that they claim to offer.

In a set of papers based on their experience of researching city dashboards and building the Dublin Dashboard, Kitchin and McArdle provided a range of critiques concerning the production and use of city dashboards (Kitchin and McArdle, 2017; Kitchin et al., 2016; McArdle and Kitchin, 2016a; 2016b). These include issues relating to epistemology, scope and access, veracity and validity, usability and literacy, use and utility, and ethics with respect to privacy, data security, and dashboard use. While each of these issues requires critical reflection and interventions, we are specifically focusing here on issues of usability and data literacy. Our focus was driven by the need to redesign and rebuild the existing Dublin Dashboard in a way that is comprehensible so that it can be meaningfully used by different types of users, including ordinary citizens, and users with limited data literacy skills.

To guide the redesign process we sought user feedback on the existing Dublin Dashboard and three other city dashboards (Hawaii, London, New York) and to construct design personas reflective of different user-types. The application of co-design practices in a smart city context (Unsworth et al., 2014) can strengthen and expand an emerging focus on HCI, particularly in the design and development of new and sustainable technologies (Rivera et al., 2015). Fortunately, there are several validated methodologies that support the implementation of user participation in systems design, such as those that are implemented in human-centered design processes (Giacomin, 2014). Furthermore, participatory design can be explored in citizen engagement situations that bring usecase scenarios to life for technology creators and help seed new technology-in-use ideas for new communities of users (Bødker, 1987; Bødker et al., 1994; Bødker and Christiansen, 1994; 2004). In this context, we focused our evaluation methodology on the combined use of inspection approaches and the evaluation of design and the use of technologies that affect groups, organizations, communities, and networks from a Computer-Supported Cooperative Work and Social Computing perspective (Nielsen, 1994; Cooper, 1999; Grudin and Pruitt, 2002; Mulder and Yaar, 2006; Bødker et al., 2012).

\section{User-Centered Design and City Dashboards}

The first and arguably most important value of Hansen's User Engineering Principles is to "know the user" (Hansen, 1971). Research has shown that the two most important issues 
for general system usability are the users' tasks, and their individual characteristics and subjective differences (Nielsen, 1994). Nielsen (1989) observed that 40 percent of external effects on system usability are due to the subjective differences that exist between users. Primarily, this means that the creators of city dashboards should always design and implement their systems with the requirements of their intended users in mind. As fundamental as this value may be, many city dashboard creators assume that they know and understand who their user will be, and what tasks they will want to perform using a dashboard without ever formally engaging with them. If they are guided at all, it is by the user requirements of the city administrators that commission the dashboard rather than the users of the dashboard, whether that be city workers or the public. This process inevitably introduces a false consensus bias, where the creators of city dashboard systems assume much more commonality between themselves and the users than exists (Ross et al., 1977).

To address false consensus bias, the creation of city dashboards needs to be more inclusive and cognizant of the knowledge and skills of anticipated user populations. Given that city dashboards are ideally created to serve a relatively broad set of users, it should be possible to quantify how different designs, visualizations, and data types can be effectively delivered to facilitate different users in making informed decisions about actionable items in everyday urban life. Therefore, city dashboard projects should aim to incorporate systems that are effective at presenting information to all types of users, regardless of ability and knowledge, via geo-specific data that are both seamlessly informative and inherently meaningful. By following this approach, the redesigned city dashboard will have higher perceived usability and utility, as the creators of the system will have an informed understanding of the different users and can, therefore, design data interactions specific to their needs. Furthermore, as is the case with many digital innovations, when a new technique or combination of techniques is conceptualized, new possibilities are suggested but not fully defined (Becker, 1982). It is widely established that city dashboards have value and an expert user base can easily be identified, but a wider reach has yet to be fully realized.

We thus make the case that city dashboards are being created sub-optimally and that they require a more informed awareness of how specific users learn, think, and answer questions using them in order to improve their design, utility, and overall user experience. By acknowledging different user-type requirements and constructively applying informed knowledge at a formative stage, it will be possible to build a city dashboard based upon evidence of data literacy and skills, showing how specific user types choose tables over graphs, words in place of numbers, or structured rather than open-ended systems (Shneiderman, 2010). In the recreation of our city dashboard, it was, therefore, important to start with an ideal scenario that could effectively represent the system user's individual requirements. However, as there was arguably no precision in the execution of this ideal in the existing city dashboard literature, formative research was undertaken to thoroughly explore and map the problem space, to analyze and gain direction within it, and to identify potential outcomes or solutions in advance of redesigning the new system.

To explore user-type requirements to guide our dashboard redesign process, we first sought to explore and identify different city dashboard user-types with the aim of creating design personas. Bødker et al. (2012) contend that personas can support and guide the design process by representing the goals, motivations, and behaviors of a target user base. Similarly, Holgersson et al. (2015) suggest that user-types, represented by personas, 
be employed to identify user-specific issues that affect perceived usability and quality. However, the use of personas can be problematic if not applied correctly. For example, design teams can have difficulty believing in personas, personas can be impersonal because it is known that they are fictional constructs, they can provide insufficient information to guide development, and irrelevant elements can be distracting (Matthews et al., 2012). These problems can be compounded further by biasing results that may appear in the creation of the personas, from the potentially innate prejudices of the researcher, and the potential exclusion of specific user groups. To mitigate the risk involved when using personas, Matthews et al. (2012) recommend that personas should not replace codesign practices without long-term strategies for user participation. Care must, therefore, be taken to explore and research these creations in analyses with actual users.

\section{Identifying User-Types}

To identify user-types we undertook a set of interviews, along with think-aloud protocols, with city dashboard users drawn from across the Republic of Ireland. At this formative stage of our project, we were seeking to generate individual feedback on existing dashboard design methods, visualization techniques, and the data content of current systems to uncover new insights that could be tested and improved in future quantitative UCD studies. The combination of interview data with protocol analysis provided us with an opportunity to open-endedly explore and reveal items of perceived quality that were not apparent in the existing literature.

Data collection and analysis took place over a period of six months. All sessions were conducted face-to-face, at locations and times that suited the individuals' requirements, and were recorded. Each session began with an explanation of the research and the session format that was to follow. Each session was scheduled for one hour and time on task generally lasted approximately 40 minutes $(\mathrm{M}=00: 41: 16 ; \mathrm{SD}=00: 12: 41)$. Interviewees included local authority staff and members of the public from the four local authorities of Dublin, Cork City, Cork County, Galway, Kildare, Limerick, and Waterford. As a broad participant group was sampled, members of the public and local authority employees were categorized by a set of characteristics that reflected city dashboard experiences and domain knowledge rather than their role. This approach reflected the user cohort in terms of user-types since novices and advanced users were found among stakeholders and the public alike.

Our target sample was 24 given that (a) the interviewees would be asked to examine four different city dashboards (counterbalanced to remove chances that the order in which the four dashboards were presented biasing the results) which required 24 orders of treatment $(4 \times 3 \times 2 \times 1)$; (b) it would be difficult to recruit double this number within the small dashboard user group available to the study through the stakeholders; (c) it was felt that the nature of the study would generate sufficient depth of knowledge that would quickly reach saturation, wherein when few additional insights would be apparent in the data (Glaser and Strauss, 2017; Fusch and Ness, 2015; Malterud et al., 2016).

Counterbalancing measures were randomly assigned to each participant in advance of their scheduled meetings. Three participants withdrew from the experiment due to scheduling conflicts and a second date could not be rearranged. The final participant pool consisted of 11 males and 10 females. The average age of the participant group was 43 years 
old $(\mathrm{M}=42.86, \mathrm{SD}=12.31)$. The education level (NFQ scale) was Advanced Certificate (level 6) $n=2$; Honors Bachelor's Degree (level 8) $n=7$; Master's Degree (level 9) $n=$ 11; Doctoral Degree (level 10) $\mathrm{n}=1$. All participants were currently working within ISCO-08 employment categories of: Technical / Engineer $n=9$; Management / Executive $\mathrm{n}=6$; Science / Medicine $\mathrm{n}=4$; and Clerical / Office $\mathrm{n}=2$.

User-types were determined by the participants' responses to a preliminary questionnaire. The initial focus of the questionnaire was on technology-in-use skills, on a scale from simple to advanced. This included a self-evaluation of digital literacies in the following areas: information technology, using the Internet, computer software, mobile applications, and social media. A Kruskal-Wallis test revealed that there were no statistically significant differences in digital technology literacy across the participants, with $\mathrm{p}>0.05$ in all areas of digital literacy. All participants were, therefore, sufficiently informed in the use of digital technology to add meaningful commentary on city dashboards.

Following this, participants were asked to quantify and comment upon their motivations to use city dashboards on a scale of casual to professional. They were also asked to identify their current knowledge of city dashboards and verbally explain their familiarity and understanding of the domain, measured from simple to complex. Based upon their self-evaluation of technology-in-use, city dashboard domain knowledge, and previous experiences with city dashboards participants were segmented into three usertypes (Cotterman and Kumar, 1989):

(1) Novice users: a novice user has some simple or mixed experiences with contemporary technology. This user-type represents new or potential city dashboard users. In most cases, they will have an egocentric, casual motivation to visit a city dashboard to learn about their local community. They have limited domain knowledge of the data presented and limited data literacy to understand visualizations presented.

(2) Primary and secondary end-users: as well as being data literate, a primary end-user is professionally motivated to use a city-specific dashboard in their day-to-day activities, primarily as a local authority employee. A secondary end-user has similar technical ability, but their motivation is contextual or intrinsically autodidactic, driven by a need to learn about the world. A secondary end-user may have knowledge of multiple city dashboard systems and wish to do simple comparative analyses on the data they access. They are advanced students of the domain who are not professionally employed to manage an urban area.

(3) Advanced users: advanced users are tech-savvy professionals with a broad knowledge of city dashboard systems and a high degree of data literacy. They have comprehensive expertise and understanding of the domain, including data and data analysis tools.

\section{Analysis of Four City Dashboards by User-Types}

After the questionnaire and preliminary interview, participants were observed interacting with selected dashboard systems via a concurrent think-aloud protocol in which they verbalized their thoughts and actions (Lewis, 1990; Kuusela and Paul, 2000). All interviews followed the same guiding question: what are the central dimensions that are relevant for the creation of a high-quality city dashboard? Participants were asked to say whatever came into their mind 
as they explored different areas of the dashboards; this included what they were looking at, thinking, doing, and feeling at that time. Interview data of this type is especially effective in a mixed-participant framework, as qualitative data is very proficient at obtaining information about the values, opinions, behaviors, and social contexts of specific user-groups (Mack, 2005). Furthermore, as a usability methodology, this type of evaluation is robust and can make use of relatively few evaluators in the detection of potential system usability issues (Nielsen, 1994). Where participants naturally finished talking, their statements were probed via interview-laddering to reveal subconscious motives (Hawley, 2009).

During each session, and to further facilitate the analysis of the collected interview data, critical incident technique (CIT) procedures were followed to collect contextual information relating to critically significant exchanges and observed behaviors that occurred during the interview. A CIT analysis is a procedure often carried out in user studies to focus upon the intentionality and implication of design strategies, identifying possible complications associated with major user-system interactions and providing a qualitative breakdown of user sentiments. Observational notes were recorded to highlight specific instants in the session that contrasted what the participants said versus what they did; specifically noting areas of the dashboard interaction where participants encounter some difficulty.

The four city dashboards selected for the analysis were Dublin, London, Hawaii, and New York (See Figure 1). These four dashboards were selected based upon several high-level criteria for the comparisons of open-data platforms. We sought archetypical dashboards that represented different approaches to dashboard design and had varying look, feel, scope, and tools. Specific considerations were data sources and veracity; variation in the visualization techniques applied; the creator's motivations; funding sources; and the classification of data. Consideration was also given for the intended target audience, the use of software licenses, interface features, data transformations, data aggregation, and the use of application programming interfaces (APIs). As far as we are aware, the dashboards involved mixed levels of user feedback in their planning and design beyond user requirements from the city office commissioning the dashboard. The Dublin Dashboard was local to some test subjects and was included in the study as it was the target of the re-design. None of the users interviewed were influential stakeholders with respect to the Dublin Dashboard. Furthermore, experiment protocol methodologies were implemented that were designed to ensure participants were providing non-biased feedback by concentrating on action and engagement rather than sentiment and opinion.

\section{Dublin (dublindashboard.ie)}

The Dublin Dashboard (NIRSA, 2014) was produced by the Programmable City project and AIRO at Maynooth University, in collaboration with Dublin City Council, Ireland. The project was created to provide Irish citizens, public service employees, and private businesses with access to thematically grouped, real-time, time-series indicator data, and interactive maps (Building City Dashboards, 2017). The Dublin Dashboard is optimized to run on a desktop web browser and consists of 11 top-level modules and numerous sub-modules, many of which are hosted by other websites. The landing page presents the user with a mix of bespoke applications developed specifically for the project and curated collections of tools and applications that were developed by other ventures. Data visualizations are presented using an SVG-based, multi-platform charting library, 


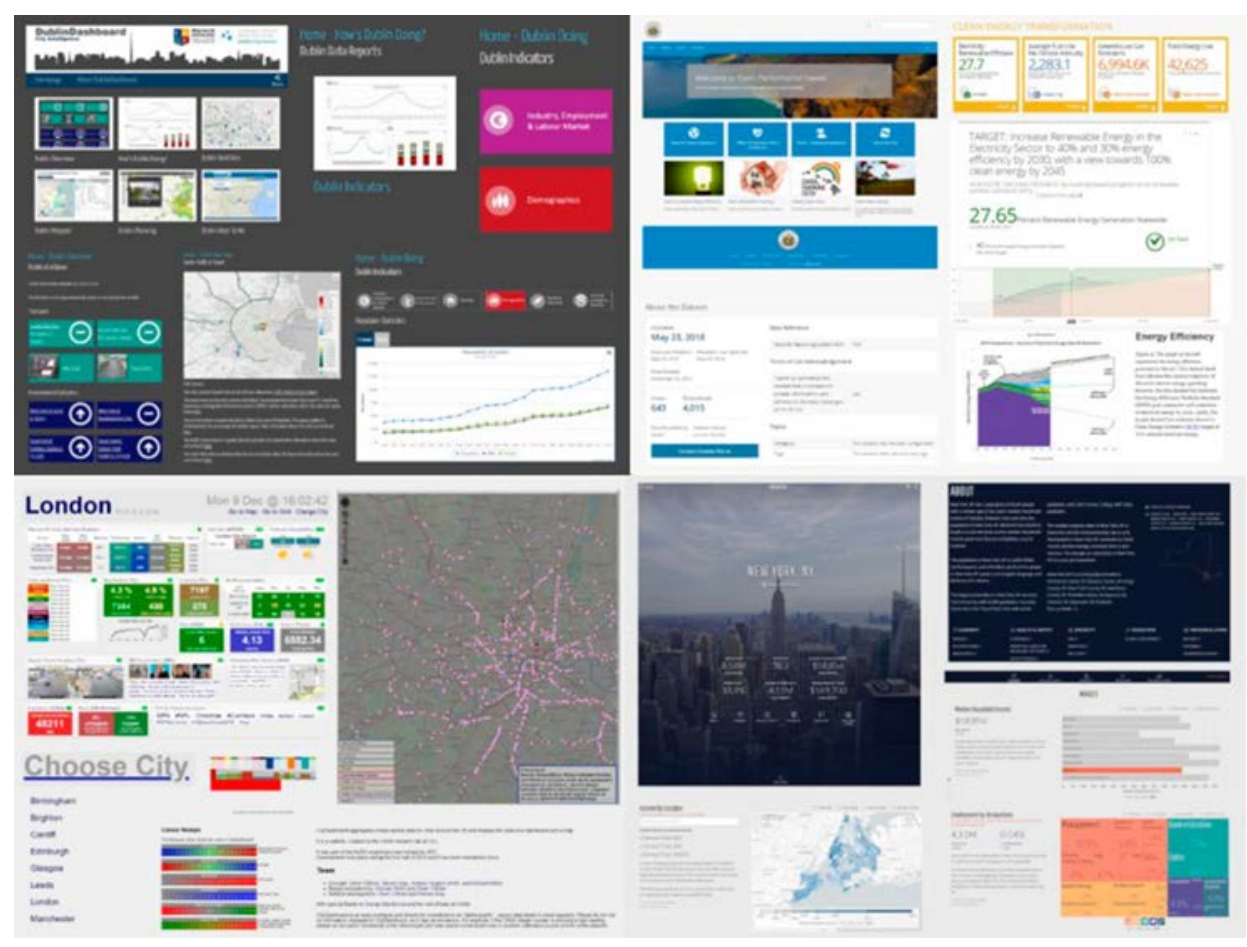

Figure 1. The archetypal elements of city dashboard websites: Dublin, Hawaii, London, and New York (left to right; top to bottom)

an open-source mapping JavaScript library, and propriety software such as ArcGIS, InstantAtlas, and Tableau. For a more in-depth account of the Dublin Dashboard design and functionality, see McArdle and Kitchin (2016b).

\section{London (citydashboard.org/london)}

The London dashboard (CASA, 2012) is an "alpha" prototype city dashboard that was created to link London data to an iPad data wall in City Hall (Smart London Board, 2013). It is an example of an "at-a-glance" city dashboard that summarizes and aggregates the quantitative real-time data for the city of London and displays this information using a modularized interface and an interactive map. The data provided in the display are sourced from a diverse set of data suppliers using APIs from JQuery, OpenLayers, and Google. Users can view real-time information about the weather, air pollution, public transport, public bike availability, river levels, electricity demand, the stock market, twitter trends, live traffic camera feeds, and the "happiness" level of the city. These data are also presented geospatially using OpenStreetMap.

\section{Hawaii (dashboard.hawaii.gov)}

The state of Hawaii launched its Open Performance Hawaii website as part of the state's IT/IRM Transformation Strategic Plan (State of Hawaii, 2014). The site is operated by 
Socrata, a government service data visualization provider that consults with governing bodies on how to build, manage, and develop digital initiatives and programs. The site allows the user to search the website, access the data catalog directly, take tutorials on how to use the data, and provides a link to a developer website to facilitate API access. The dashboard presents the public with a broad set of information via data visualizations, for example, budget and economy, education, healthcare and seniors, energy, agriculture and environment, public safety, and open government. Users can monitor the state's performance through the comparison of historic and current data as key performance indicators (KPIs). Linked beneath these indicators are more in-depth data, presenting graphical visualizations of annual trends and links to data sources.

\section{New York (datausa.io/profile/geo/new-york-ny)}

The New York dashboard (Data USA, 2014) is part of the larger "Data USA" project that was developed by the MIT Media Lab. The New York section of the Data USA website presents users with data on the state, the metropolitan area, the city, and other small areas within the New York boundary. For the study, only city-level data were displayed. The New York City landing page displays six static statistics: population, median age, median household income, poverty rate, number of employees, and median property values. Below are six sections, each representing specific thematic categories. Each subcategory has a short descriptive sentence supported with a data visualization. The individual data sources are accessible from their multiple sources. The data on the site can be accessed via an API and each visualization can be saved, shared, or compared to other locations in the USA.

By applying think-aloud protocols to these four dashboards and analyzing with both content analysis and critical incident techniques it was possible to observe the cognitive processes associated with dashboard system interaction and quantify specific elements of usability and perceived quality. A content analysis (CA) is a research method for studying communication artifacts and making replicable and valid inferences through the interpretation and coding of transcripts (Denzin and Lincoln, 2008). The CA explored the communication of city dashboard quality artifacts and examined patterns in user communications in a systematic manner. From the transcripts, coherent thought-units were extracted, where a single thought-unit represented a contiguous or holistic statement (Hatfield and Weider-Hatfield, 1978). Each thought-unit was then reviewed for further division into coherent single statements (ss) as the participant pool exhibited different experiential quality criterions within individual thought units. These single statements were then matched for semantic similarities, removing any further redundancies. An affinity-diagramming workshop was conducted by three project researchers to group semantically similar words or phrases under a collective category or to split categories into different elements using human insight and subject matter knowledge (Rosenfeld and Morville, 2002). This process generated hierarchical content categories in a bottom-up procedure (See Figure 2) (Beyer and Holtzblatt, 1999).

\section{User-Type Similarities and Differences}

The CA results presented and categorized perceptions of quality and usability and are indicative of the collective attributes of the four city dashboards (See Figure 3). Following the three-tier structuring of the CA, the CIT analysis results were organized within Tier 2 

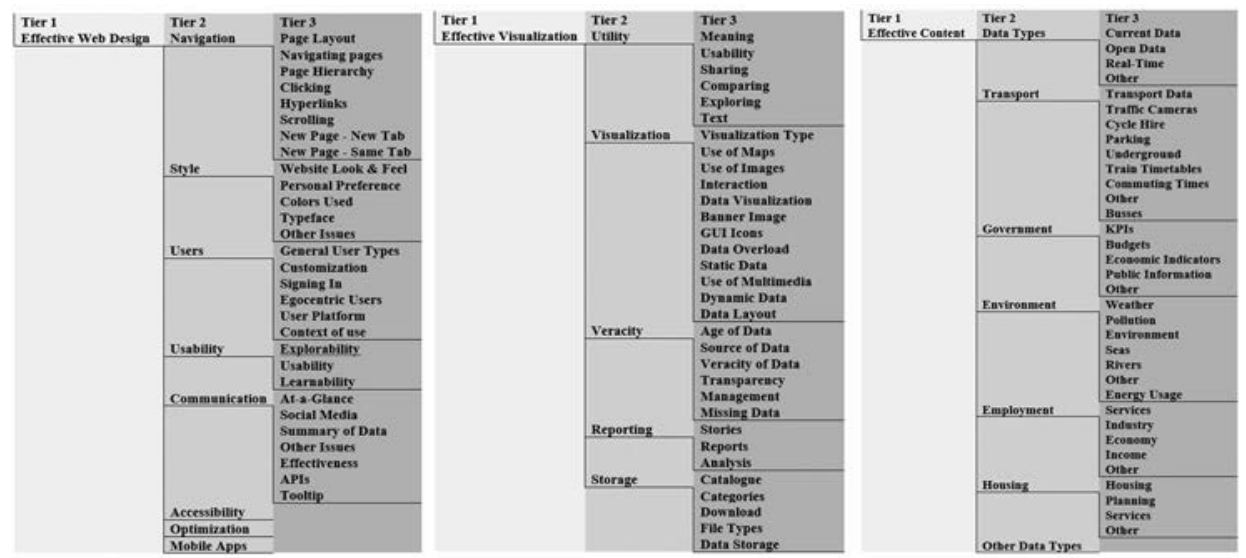

Figure 2. Three-tier hierarchical representation of content analysis categories

of the CA to represent the high-level differences between the user-types' thoughts and attitudes (See Figure 4). The categories of the CA and CIT for each user-type emphasized how website design, data visualization, and dashboard content were influential to the evaluations made by the users.

These results were analyzed in order to explore how specific aspects of city dashboards affected the users' perception of the quality of website design, data effective visualizations, and data content.

\section{Content Analysis}

The CA highlighted that there were subtle similarities and differences between the concerns of the different user types, for example, between the advanced users and end-
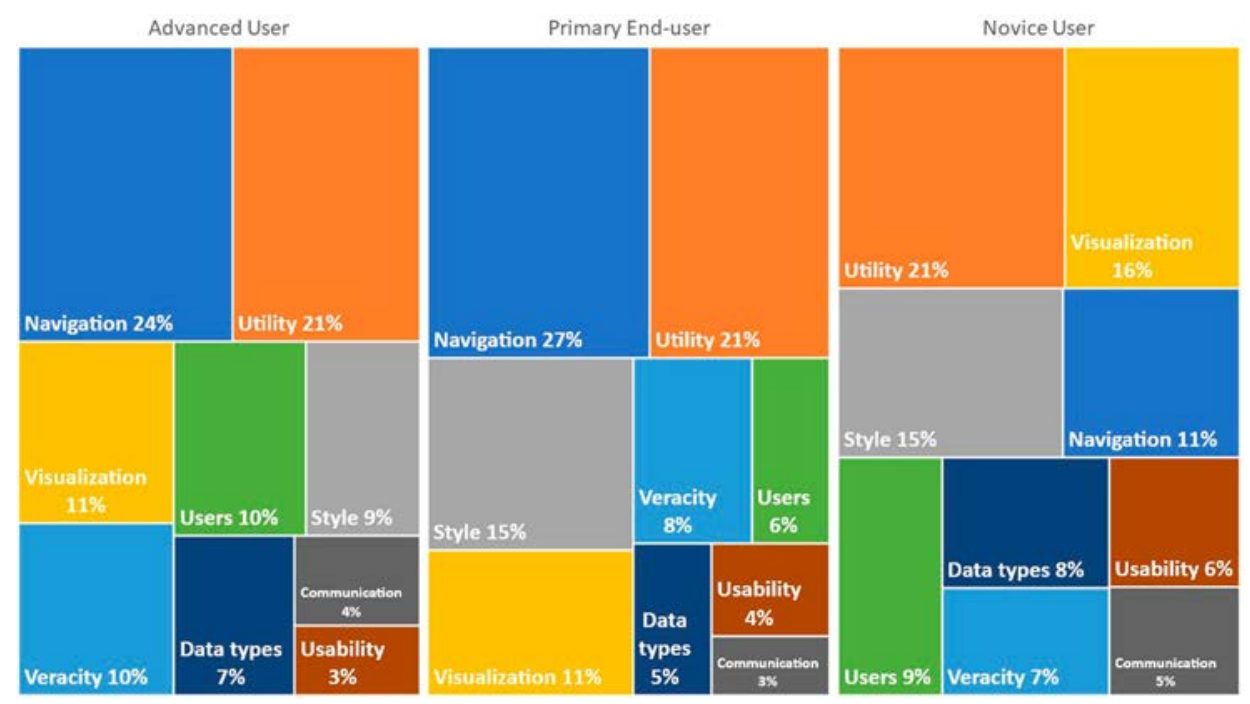

Figure 3. Content analysis treemap for the top 9 Tier 2 categories identified by user-type; novices (ss = $740)$, end users (ss $=2465)$, and advanced users $(s s=1610)$ 


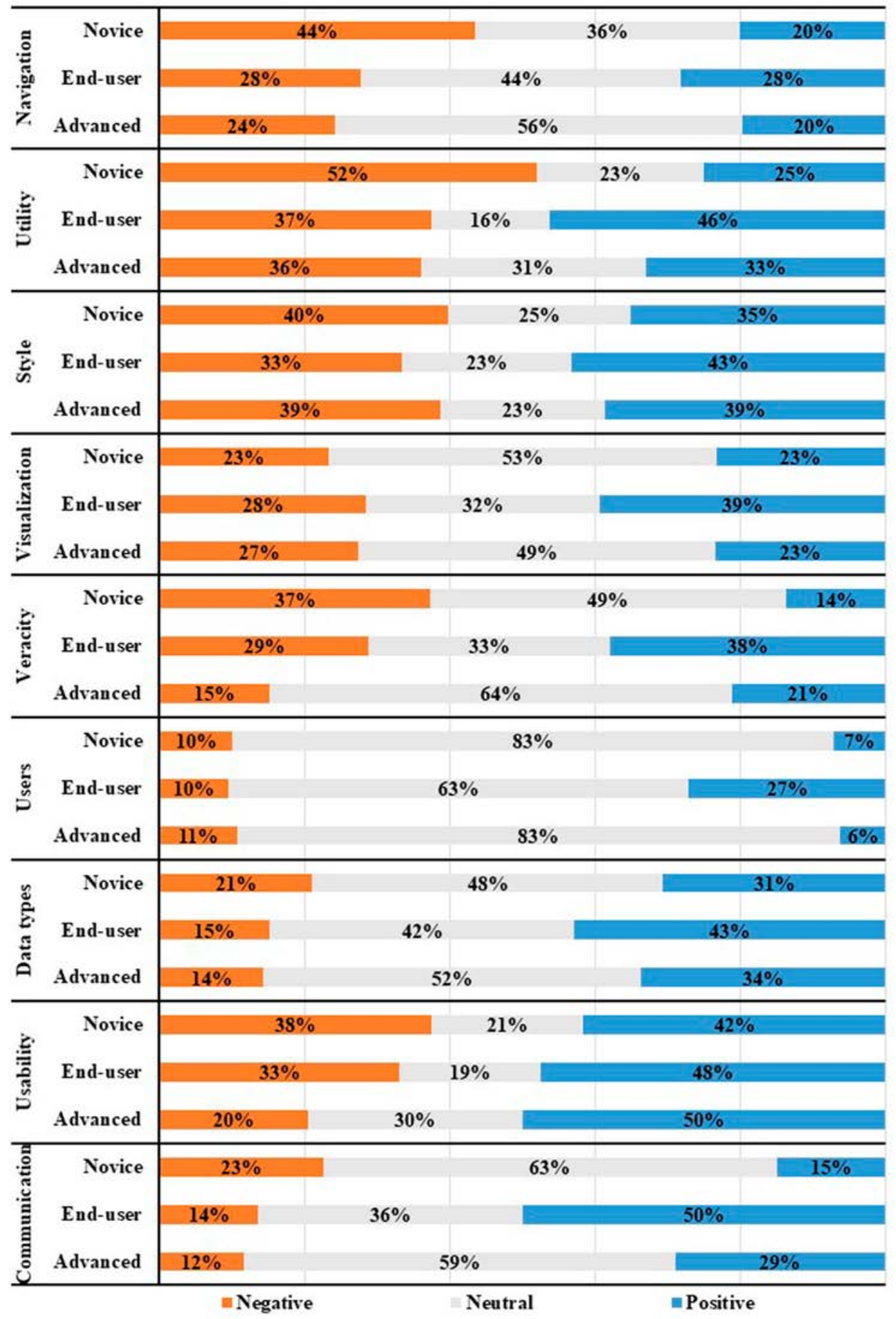

Figure 4. Diverging stacked bar chart showing CIT for the top 9 Tier 2 CA categories identified by usertype 
users. This was indicative of the professional experiences of these user groups, affording them familiarity with using city dashboards. Specifically, the CA revealed that for the advanced users and end-users alike, the navigation of a city dashboard was an important area of concern (See Figure 3). For novice users, navigation was focused upon somewhat less but was still highly commented upon. This highlighted the apparent effects of city dashboard domain knowledge on expectations of quality in the logical navigation patterns for users with professional or somewhat more task-driven or focused motivations. In the same context, the general style, look, and feel of the city dashboard was of greater importance to both the end-user and the novice than that of the advanced user. This observation was perhaps related to broader website experiences, as these types of users interact with various websites daily and have high expectations of the diverse usability and quality factors of modern website design. Some variations in the user-type analysis were also observed in the evaluation of data veracity, although its occurrence in the CA was considerably lower than expected. It was, in this instance, the advanced users who expressed concern over how reliable the data were that were being presented to them. This is reflective of the novice user-type being unable to find innate meaning or usefulness in the data presented to them.

The CA results present similarities and variations in user perceptions of quality and usability and are indicative of the nuanced relationships between the types of users identified in the segmentation we applied for user-types. These differences were noted between user-type results, emphasizing how each of the dashboards was perceived to be of relevance and use to the people currently engaging with them. These similarities and differences support observations made by Zhu et al. (2015), where the "digital divide" presents differences between types of users. By observing and noting these differences in our redesign process, we can help support the creation of meaningful digital interventions that facilitate trust in civil engagements performed by both citizens and local authorities (Corbett and Le Dantec, 2018).

\section{Critical Incident Technique}

The CIT analysis was organized with respect to Tier-2 CA categories, as per Figure 2. The analysis highlighted that there were just three critical incident areas that were commonly experienced across the selected city dashboards (See Figure 4). With respect to data visualization, all users experienced similarly negative incident outcomes ( \pm 5 percent). This trend was also observed for critical incidents that involved empathy for different types of users and their individual requirements, although the number of negative outcomes was considerably fewer (10-11 percent). Similarly, for dashboard usability, overall positive sentiment was expressed by all user-types ( \pm 8 percent).

Across the other categories, there were notable variances in the positive and negative outcomes of critical incidents experienced by user-types. The end-users and the advanced users each experienced a similar number of negative outcomes for navigation ( \pm 4 percent), forgiving several fundamental website navigation issues caused by poor page layouts and information architectures. These groups of users also agreed upon negative outcomes for the types of data presented on the dashboards ( \pm 1 percent), as well as how effective the dashboards were at communicating and reporting data ( \pm 2 percent). With regard to positive critical incident appraisal, both the advanced and end-users agreed that the style of the 
dashboards was acceptable ( \pm 4 percent). A commonality between end-users and novices was seen in the negative appraisal of issues relating to usability, where a high number of negative usability outcomes were reported ( \pm 5 percent). Novices and advanced users described the same positive opinions towards the navigation of dashboards and the effectiveness of the visualizations used; shared very similar sentiments for empathy towards other user needs ( \pm 1 percent), and were generally happy with the different types of data being displayed in both real-time and historic forms ( \pm 3 percent). Finally, novices and advanced users were also more critical of the style choices made across all dashboards ( \pm 1 percent).

Different user-types also presented with notably dissimilar sentiments towards critical incidents. The novice users experienced a higher number of negative critical incident outcomes for city dashboard navigation (44 percent), where many difficulties were experienced when navigating around the individual pages, the data modules they were presented with, and the user interface. Furthermore, novice users struggled to direct themselves around the dashboards, being regularly confounded by unclear primary navigation options and indistinguishable secondary navigation schemes, such as breadcrumbs. Novices also failed to find utility in the types of data presented to them (52 percent). This was particularly prominent in the clarity in presenting facts and statistics for reference and analysis as well as when meeting the users' needs and delivering the expected data. With regards to dashboard style, the novice users were also less inclined to express positive sentiment towards the look and feel of the individual dashboards (35 percent) than the other user-types. They were also more critical of incidents surrounding the types of data that they were presented with, particularly the effectiveness of the content (21 percent). Finally, the novice users experienced more negative incidents relating to the way dashboards communicated and reported data (23 percent). In particular, these users struggled to make sense of the data as well as the broader contextual meaning of the data they were being presented with.

The primary and secondary end-user cohort was observed being more forgiving when navigating the different dashboards (28 percent). Primarily, incidents involving the individual page layouts and the arrangement of data within modules on the dashboards were praised more openly than the other user-types. Furthermore, the occurrence of positive critical incident outcomes for visualization methods applied to the different dashboards was resolved with a much more optimistic result (39 percent). This was also true regarding this user type's empathy for other users ( 27 percent) and their approval of the different types of data being displayed (43 percent). The end-users were also less critical over the effectiveness of dashboard-style choices, favoring function over form (33 percent).

The advanced users were inclined to express similar critical incident outcome traits to both novice and end-user user-types alike, as outlined above. However, this user cohort provided considerably fewer criticisms about dashboard usability (20 percent), choosing instead to focus on other areas of city dashboard effectiveness.

Significantly different critical incident outcomes were observed for the CA categories of utility, veracity, and communication, where unique observations were made between each of the user-types. Starting with the apparent utility of the data, the clarity of its meaning and its actionable applications in the real world, the end-users resolved more critical incidents with positive outcomes (46 percent). This was followed by the advanced users (33 percent), and finally the novice user-type (25 percent). Primarily, the novice users saw 
very few actionable use cases when viewing city data and the end-users recognized the different sources from previous experiences of interacting with data. When regarding the efficiency of how well the dashboards communicated their data, the primary and secondary end-users were again more inclined to experience positive critical incident outcomes over the other user-types (50 percent). The advanced user's sentiments fell in-between the end-user and novices (29 percent). The novice user's evaluation of critical incidents in this category was not only inclined to be more critical but was also less likely to resolve incidents with any certainty (15 percent). This was again observed as a familiarity issue, where end-users were well versed in reading such communications on a regular basis. Finally, the CA category of veracity received mixed sentiments across all user-types. When resolving critical incidents relating to the age and source of the data, the novice users were unable to find or were uncertain about who the data were being provided by (37 percent), which was generally expressed as a distrust of how the data were collected and when. The novices were also inclined to resolve fewer incidents with a positive outcome as they could not gather information or process meaning easily (14 percent). In comparison, the end-users were able to garner the most positivity from the veracity of the data presented to them (38 percent). However, they were also able to determine a relatively similar number of negative elements also as they knew which data providers to have confidence in and who not to trust (29 percent). The advanced users were less inclined to experience negative data veracity incidents than the other user-types (15 percent) and were similarly less likely to offer positive sentiments than the end-users (21 percent).

\section{Creating Design Personas}

To communicate user-type requirements, the collected data were used to create targetedscope user personas to help direct formative system design and development and communicate the innate variances in dashboard designs for different types of user engagement (Pruitt and Grudin, 2003; Idoughi et. al, 2012; Holgersson, 2014). To begin the persona-building process, the uniquely identifiable, segmented elements of the user observations were collated and organized into their respective user-types. During this process, specific user-type attributes formed clusters of personable characteristics that related directly to the completed research activities. As these clusters of data began to grow in similarity, they were combined to remove repetition and, similarly, if newly shaped characteristics were deemed less important for city dashboard systems, they were removed.

As distinct personable features emerged for user-types from the data, extra details were embroidered into the process to make the personas appear more realistic, believable, and memorable, while avoiding stereotyping the user. In this process, a balance was required between adding unnecessary details that contributed no meaningful consequences to the design process and creating memorable characteristics that a project team could use when building a user-centered city dashboard. Furthermore, Blomquist and Arvola (2002) suggest that when using personas, the design team must include members who are familiar with or involved in the personas-creating process. These experienced persons should then guide the team in the creation of persona and use-case scenario-driven design strategies. The personas presented in Figures 5, 6, and 7 were created by making informed decisions 


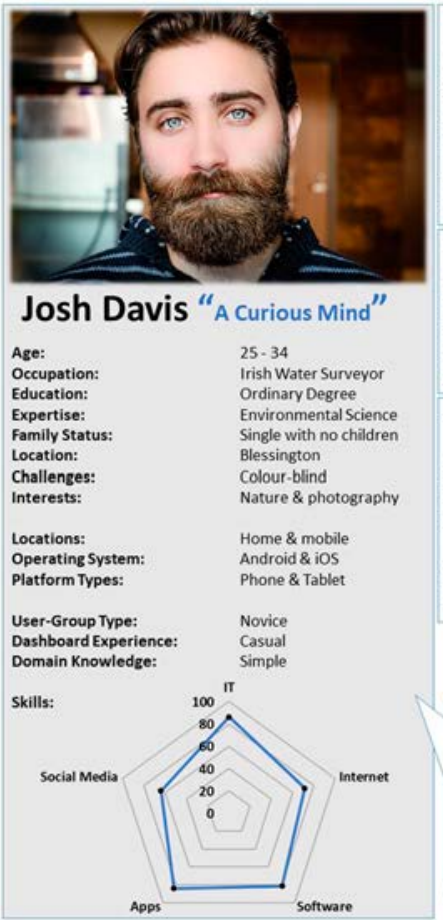

Experience \& skills:

Josh is curious, so he has visited the dashboard once or twice before. He originally found the dashboard through Google after seeing a tweet about it. He is somewhat familiar with looking at thematic data having used it for his undergraduate degree.

- Referrer: Search engine

Introduction touchpoint: Twitter

\section{Interacting with Josh:}

osh explores the site to see what the dashboard can tell him about his city. The stories are really interesting to him, because they show how the data is meaningful to his community. He explores other themes based upon what he learns from his interests.

\section{Typical Tasks for Josh:}

\section{osh usually lands on the home page from Google. He} likes to browse the environmentally themed pages to help him make plans for photography trips around Dublin.

Josh is also interested in transport data because he has to drive to multiple sites across the city throughout the week for work.

Josh's Story: "I hate to always come back to aesthetics, but..."

Josh has had casual experiences with city dashboards in the past and has a simple understanding of the city dashboard domain. He does not have any knowledge about some of the more specific data sets or the potential meaning of city data in a larger context. He struggles with the application of such data for actionable urban activities and the broader concepts and relationships that exists between data themes. However, Josh is a curious semi-professional who is educated and tech-sary. An individual who has the capacity to grasp the use of city dashboards, but only in certain circumstances. Any anxieties that Josh capas may expere

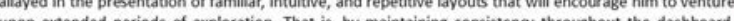
Further Furthermore, the stories that the dashboard presents help Josh to contextualise the data and build upon his current knowledge of data relationships.

Figure 5. Novice user persona "Josh Davis"

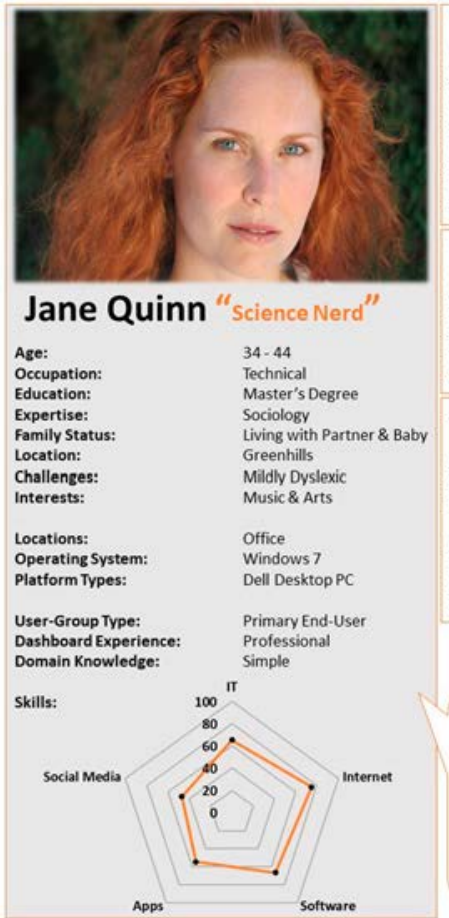

\section{Experience \& skills:}

lane uses the dashboard regularly at the local county council (CC) where she works. She is familiar with data stories and thematic data content. She regularly explores new data use-cases, routinely following links to data sources to check their veracity, and makes use of visualisations in her monthly reports.

Introduction touchpoint: Data Coordinator

\section{Interacting with Jane:}

lane visits the site with specific tasks and goals in mind. She learned about the site from a training session that she attended through work. Jane knows that if she has to undertake a new line of enquiry, she can use the "Tasks" page to learn new skills.

\section{Typical Tasks for Jane:}

ane has the site saved in her "Work" bookmarks folder in Chrome. She often keeps a tab open for reference throughout the day.

Jane's job requires her to write monthly reports about housing completion rates in the local CC area. She also needs to compare her $\mathrm{CC}$ to other local authorities with similar population sizes.

\section{Jane's Story: "Crisp and clean designs with up to date data."}

Jane has professional experience with city dashboards, but has a simpler understanding of the city dashboard domain as a whole. Jane is familiar with most city-specific systems and has used some of the data tools. However, she does not access in-depth data on a regular basis. Jane has regular tasks to perform for a city-specific location. This gives her explicit expectations of a system and a comprehensive understanding of task-driven applications. These motivations give her an advantage over new users, but she still has difficulty learning new menu structures and navigating to data-sets on unfamiliar or overty complex pages. Jane avercomes these issues by systematically following menu patterns and page structures, She appreciares consisent and professional de visulisation methe data visualisation methodologr. These suppont her in creating patterns-of-use that are easy to remember online training and help-pages, but only if they are quickly accessed, well-organised, and easy-to-use.

Figure 6. Primary end-user persona "Jane Quinn" 


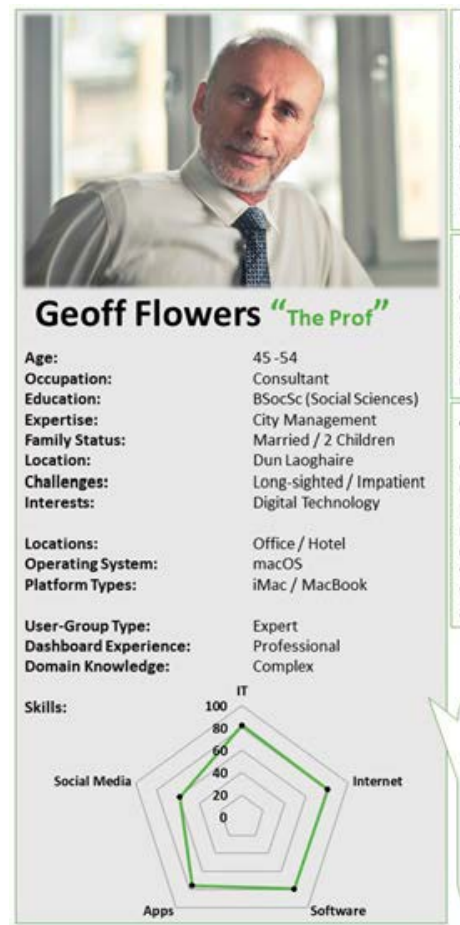

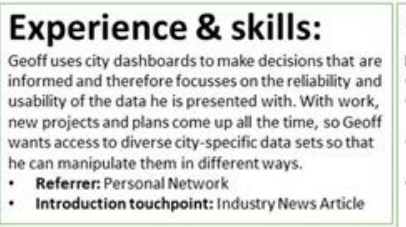

Interacting with Geoff:

Geoff accesses multiple dashboards from work and sometimes when travelling abroad. He needs to be able to view and compare different data types as well as being able to explore new data sets as they are made available, often on a global scale.

Typical Tasks for Geoff:

Geoff typically needs to communicate and comment upon high impact projects based upon his research. He is familiar with multiple data types and uses multiple data comparison tools. He wants to compar cities in his country with other European locations, which isn't really facilitated elsewhere. Geoff needs and convey his findings to an often-broad audience.

\author{
Geoff' Situation: \\ His Goals / Motivations \\ Looking for task and tool-based options. \\ Wants to answer specific questions about places \\ and social processes. \\ Needs tools with multifunctionality and \\ extended capabilities. \\ Needs to communicate and explain his. \\ understanding of the data.
}

Geoff's Story: "Entice me to dig deeper and do more."

Geoff considers himself as an advanced user. He has had many previous professional experiences with city dashboards and has a complex knowledge of the different types of data available in the city dashboard domain. Geoff's knowledge and familiarity of complex data is what motivates him to accomplish his goals efficiently, in terms of both general day-to-day and more specific tasks. Geoff expects a city dashboard to efficiently, in terms of both general day-to-day and more specific tasks. Geoff expects a city dashboard to
possess a rapid response time, have a diverse and up to date data set, provide functional tools, and he expects to be able to acquire and navigate to specific data sets within relatively few steps. For Geoff, when carrying out tasks that are performed regularly, it is necessary for him to create familiar procedures that can be executed and accessed via shortcuts, through an advanced menu system, such as accessing specific data resources and tools or repurposing data visualisations for custom applications, such as for professional presentations and the likes.

Figure 7. Advanced user persona "Geoff Flowers"

about the potential characteristics of a user. However, it should be clearly stated that a user-type is not the same as a persona, and vice versa, as a persona is created to be a more personal representation. These personas are representative of fictional people, but they were created from the data collected from and about real people. Therefore, from the study of the city dashboard presented, it was possible to develop personas to remind our city dashboard team and stakeholders for whom they are creating their product. This technique instilled a sense of empathy for our identified users by contextualizing the results gathered and bringing to life the potential user in the form of an imaginary person.

One of the most apparent benefits of using personas within our project has been that they have facilitated the use of precise vocabulary for describing users' motivations and requirements and this, in turn, has helped to focus the project teams' efforts towards creating a city dashboard that is accessible to the three user-types. For example, when referencing specific user requirements or individual user differences in a design context, the user-type is referred to by name, i.e., "What would Josh/Jane/Geoff do?"; "How would this design decision affect Josh/Jane/Geoff?" By framing a design objective around a specific person, the project breaks self-referential thinking and removes reliance on personal opinions, shifting dialog away from personal opinions of user needs to the needs identified in our user study and informing which features need implementation and prioritization (Harley, 2015). This type of practice has changed how the design and software development team thinks about and relates to the dashboard by innately building empathy within the project team for how real people will potentially use the new system in 
the future. Furthermore, because specific guiding principles from validated studies were used to create the dashboard personas, they inherently dictated restrictions on the design process. That is, in the creation and adaptation of personas in this specific use context, all key stakeholders and team members within the project were required to use them. By including all project team members in the application of personas, the function of user testing and user empathy has become more apparent for future city dashboard evaluation tasks and iterative design practices.

\section{Dashboard Design Recommendations}

A direct result of the user-type research and the adoption of personas within the project team has been a fundamental restructuring of the website that avoids a one-size-fits-all design. The new dashboard integrates descriptive (explanatory), systematic (exhibitory), and analytical (exploratory) data sections. Different user-types are now directed to four different paths through the dashboard content, organized by "Stories" (novice user), "Tasks" (end-user), "Tools" (advanced users), and the data that underpin all these sections can be found in "Themes" (for all user-types). Essentially, what has now been presented to the user conforms to their identified data literacy and competencies (See Figure 8).

The data stories section presents visualizations that are accompanied by textual information that guides the interpretation of the data and links related data together to create a narrative that explains a domain. The aim is to provide explanatory data analytics and deliver practical insight into actionable data. Although improving data literacy and understanding is key, it is also essential for novices to understand the meaning of data in relation to the city around them. As such, a housing data story sets out the story of housing development over time, with the narrative heavily supported by several interactive data visualizations that illustrate key trends.

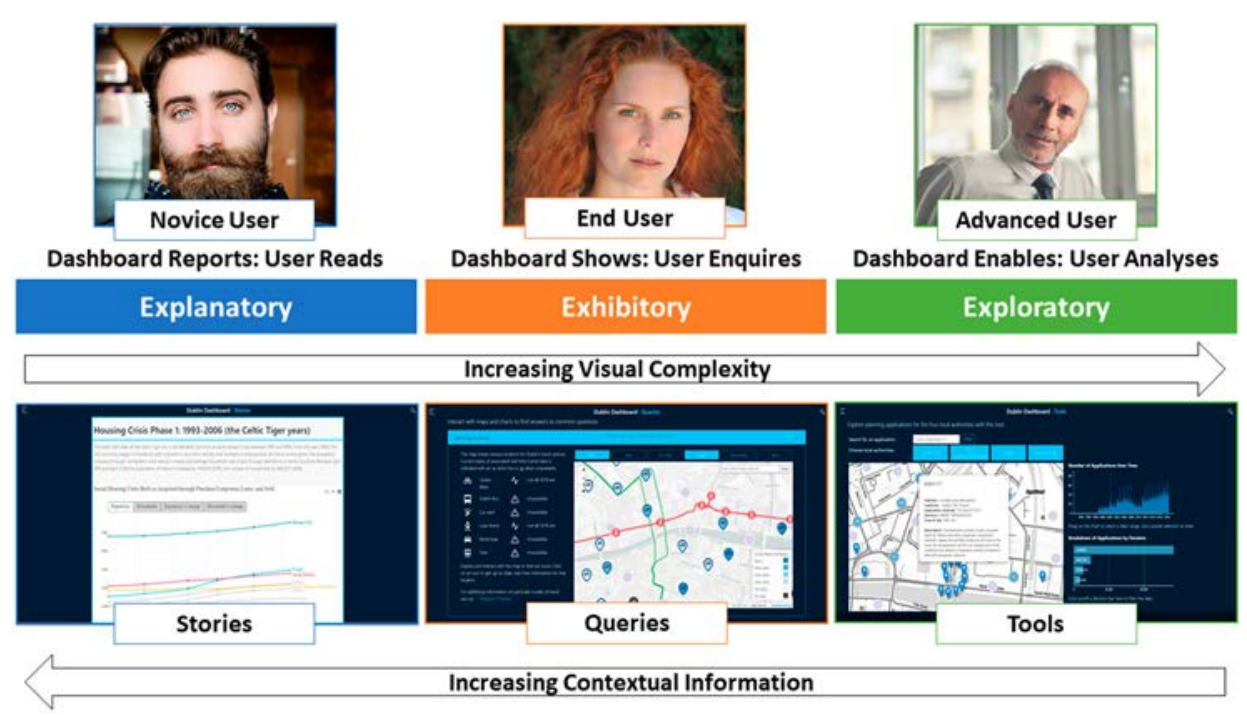

Figure 8. An overview of user-type research as applied to city dashboard redesign and development 
For end-users, our analysis suggests that an exhibitory approach to city data would facilitate these users in their day-to-day dashboard interactions. They possess a level of data literacy that removes the need for explanatory supports, but their work is taskorientated rather than exploratory. The tasks section thus enables the user to ask questions and query the data to accomplish basic tasks. For example, the "Homes and Housing" query presents historical data relative to a user-defined time and location, facilitating the user in answering specific questions, such as "What planning permissions were given in my community last year?" and constructing bespoke text and visualizations in response.

During our interviews, the advanced users indicated that they wanted direct access to the raw, underlying data of the city dashboards they encountered, plus access to advanced analytic tools. They were motivated to look past the contextual information and dive deeper into an exploratory, self-led analysis of the data. Advanced users want to apply their knowledge and experience to understand associations and relationships and obtain new insights, but also examine the data for errors and anomalies, explore outliers, and test new assumptions. The tools section is, consequently, designed to enable advanced users to build their own tasks, to access and query the data directly, and to undertake advanced data analytics, including statistical testing and comparative studies. These advanced tasks are beyond the competencies of most novice and endusers, who only encounter this section if they stray from their designated path, though with experience and training they might progress to actively using these tools, and the dashboard is incorporating educational tools into its design to help facilitate such a transition.

In addition, given the emphasis placed on data meaning and usability, visualizations, and website styling by all users, much effort has been invested in the design and lookand-feel of the dashboard as a whole for all users, as well as the creation of individual sections and more specific data visualizations, with the aim of aiding data literacy and improving the interpretation of dashboard elements from multiple user perspectives. Although we did not validate our newly created personas in this study, we are presently user testing the new dashboard, where participants identify themselves as specific usertypes. Furthermore, iterations of this research will also include the evaluation of these pages from the perspective of user-types, in a stratified task-based analysis.

\section{Conclusions}

If city dashboards are going to fulfill the smart city and open government ambitions to enable transparency of city administration and create a more informed populace, they need to be designed in a way that fulfills user expectations and can be used by citizens with varying domain knowledge and data literacies. This requires dashboards to be created via a UCD approach. In our study, usability engineering techniques were applied to tackle the shortcoming of a homogenous usability evaluation using traditional task-based evaluation practices (Nielsen, 1994). Although these techniques have been previously explored (Máchová and Lněnička, 2017; Kapoor et al., 2015), our principal focus was the evaluation of city dashboards from a variety of user perspectives. Furthermore, while there have been previous studies examining dashboard and open-data portal usability (Attard et al., 2015; Kubler et al., 2016), there are very few examples of user-centered 
investigations that are undertaken to empathize with the non-specialist-users' perception of usability and quality. We have thus sought to fill this lacuna by conducting an empirical investigation of user experience, user-types, and personas to provide an evidence base for guiding city dashboard design and implementation.

The next phase of our research is to undertake a further round of quantitative user evaluation of the redesigned dashboard and to gather feedback from different usertypes on the division into stories, tasks, tools, and themes pathways that can inform the ongoing iterative process of reflection and refinement of the design. Given the growth in city dashboards globally and the general lack of broad user engagement in the requirements and design process, additional task-based studies are required to further consider user-type and personas in producing high quality and effective systems. Although the process of getting to "know the user" may appear as a never-ending cycle, every step towards identifying, understanding, and empathizing with users is a step closer to successfully implementing dashboards with strong usability and utility for all users.

\section{Disclosure Statement}

No potential conflict of interest was reported by the authors

\section{Funding}

The research in this paper was funded by the Science Foundation Ireland (SFI), under the Investigators' Award Program [award number 15/IA/3090].

\section{Notes on Contributors}

Gareth W. Young is a postdoctoral researcher on the V-Sense project, School of Computer Science and Statistics, Trinity College Dublin, Ireland.

Rob Kitchin is Professor of Human Geography in the Maynooth University Social Sciences Institute and Department of Geography at Maynooth University, Kildare, Ireland.

Jeneen Naji is Digital Practice Coordinator and Digital Media Faculty in the Department of Media Studies at Maynooth University, Kildare, Ireland.

\section{ORCID}

Gareth W. Young (D) http://orcid.org/0000-0002-8763-4668

Rob Kitchin (D) http://orcid.org/0000-0003-4458-7299

Jeneen Naji (1) http://orcid.org/0000-0002-7479-0143

\section{References}

J. Attard, F. Orlandi, S. Scerri, and S. Auer, "A Systematic Review of Open Government Data Initiatives," Government Information Quarterly 32: 4 (2015) 399-418.

H. S. Becker, Art Worlds (Berkeley: University of California Press, 1982).

R. D. Behn, The Performance Stat Potential: A Leadership Strategy for Producing Results (New York: Institution Press, Ash Center 2014).

H. Beyer and K. Holtzblatt, "Contextual Design." Interactions, 6: 1 (1999) 32-42. 
Å. Blomquist and M. Arvola, "Personas in Action: Ethnography in an Interaction Design Team" Proceedings of the second Nordic conference on Human-computer interaction, (2002) 197-200.

S. Bødker, Prototyping Revisited: Design with Users in A Cooperative Setting, Report of DAIMI Series 233 (Aarhus: Aarhus University, 1987) <www.researchgate.net/profile/Susanne_Bodker/ publication/295899948_Prototyping_Revisited_-_design_with_users_in_a_cooperative_setting/ links/576b6fac08ae6e772780bc4a.pdf> Accessed August 12, 2019.

S. Bødker, E. Christiansen and M. Thüring, A Conceptual Toolbox for Designing CSCW Applications, Report of DAIMI Series 489 (Aarhus: Aarhus University, 1994) <https://www.researchgate.net/ profile/Susanne_Bodker/publication/228800099_A_conceptual_toolbox_for_designing_CSCW_ applications/links/09e4150d1cf59f40ea000000.pdf > Accessed August 12, 2019.

S. Bødker and E. Christiansen. Scenarios as Springboards in The Design Of CSCW, Report of DAIMI Series 488 (Aarhus: Aarhus University, 1994) <https://tidsskrift.dk/daimipb/article/download/ 6982/5946> Accessed August 12, 2019.

S. Bødker and E. Christiansen, Designing for Ephemerality and Prototypicality, paper presented at DIS'04, Cambridge, August 1-4, 2004.

S. Bødker, E. Christiansen, T. Nyvang and P. O. Zander, "Personas, People and Participation: Challenges from The Trenches of Local Government," paper presented at the Twelfth Participatory Design Conference: ACM Research Papers Vol 1, Roskild, August 1216, 2012.

D. T. Bourgeois and T. A. Horan, "A Design Theory Approach to Community Informatics: Community-Centered Development and Action Research Testing of Online Social Networking Prototype," The Journal of Community Informatics 3: 1 (2007) Retrieved from http://jat.gws.uky.edu/index.php/ciej/article/view/308.

Building City Dashboards Project website, "Homepage" (2017), <http://dashboards.maynooth university.ie> Accessed January 11, 2019.

CASA, "London Dashboard" (2012) <http://citydashboard.org/london/> Accessed October 11, 2018.

A. Cooper, The Inmates Are Running the Asylum: Why High-Tech Products Drive Us Crazy and How to Restore the Sanity (Indianapolis: Sams, 1999).

W. W. Cotterman and K. Kumar, "User Cube: A Taxonomy of End-Users," Communications of the ACM 32: 11 (1989) 1313-1320.

E. Corbett and C. A. Le Dantec, "Going the Distance: Trust Work for Citizen Participation," paper presented at the CHI Conference on Human Factors in Computing Systems, Montréal, April 2126, 2018.

Data USA, "New York, NY" (2014) <https://datausa.io/profile/geo/new-york-ny/> Accessed October 11, 2018.

N. K. Denzin and Y. S. Lincoln, Collecting and Interpreting Qualitative Materials (Thousand Oaks: Sage, 2008).

K. C. Desouza and A. Bhagwatwar, "Technology-Enabled Participatory Platforms for Civic Engagement: The Case of US Cities," Journal of Urban Technology 21: 4 (2014) 25-50.

P. I. Fusch and L. R. Ness, "Are We There Yet? Data Saturation in Qualitative Research," The Qualitative Report 20: 9 (2015) 1408-1416.

C. P. Geiger and J. von Lucke, “Open Government And (Linked) (Open) (Government) (Data)," JeDEM-eJournal of eDemocracy and Open Government 4: 2 (2012) 265-278.

J. Giacomin, "What is Human-Centered Design?" The Design Journal 17: 4 (2014) 606-623.

B. G. Glaser and A. L. Strauss, Discovery of Grounded Theory: Strategies for Qualitative Research (Abingdon: Routledge, 2017).

J. Grudin and J. Pruitt, "Personas, Participatory Design and Product Development: An Infrastructure for Engagement," paper presented at Participatory Design Conference, Malmo, 23-25 June, 2002.

W. J. Hansen, "User Engineering Principles for Interactive Systems," Proceedings of the Fall Joint Computer Conference (1971) 523-532.

A. Harley, "Personas Make Users Memorable for Product Team Members" (2015) <www.nngroup. com/articles/persona/> Accessed March 9, 2018. 
J. Hatfield and D. Weider-Hatfield, "The Comparative Utility of Three Types of Behavioral Units for Interaction Analysis," Communications Monographs 45: 1 (1978) 44-50.

M. Hawley, "Laddering: A Research Interview Technique for Uncovering Core Values" (2009) $<$ www.uxmatters.com/mt/archives/2009/07/laddering-a-research-interview-technique-for-unco vering-core-values.php> Accessed September 20, 2017.

P. Hemmersam, N. Martin, E. Westvang, J. Aspen, and A. Morrison, "Exploring Urban Data Visualization and Public Participation in Planning," Journal of Urban Technology 22: 4 (2015) $45-64$.

J. Holgersson, "User Participation in Public e-Service Development: Guidelines for Including External Users," (PhD diss. University of Skövde, Skövde, 2014) <www.diva-portal.org/smash/ record.jsf?pid=diva2\%3A760905\&dswid=orcid.widget $>$ Accessed September 20, 2017.

J. Holgersson, B. Alenljung, and E. Söderström, "User Participation at a Discount: Exploring the Use and Reuse of Personas in Public Service Development," paper presented at the European Conference on Information Systems, Münster, May 26-29, 2015.

D. Idoughi, A. Seffah, and C. Kolski, "Adding User Experience into The Interactive Service Design Loop: A Persona-Based Approach," Behavior and Information Technology 31: 3 (2012) 287-303.

K. Kapoor, V. Weerakkody and U. Sivarajah, "Open Data Platforms and their Usability: Proposing A Framework for Evaluating Citizen Intentions," paper presented at the Springer conference on e-Business, e-Services, and e-Society, Delft, October 13-15, 2015.

R. Kitchin, T. Lauriault, and G. McArdle, "Knowing and Governing Cities Through Urban Indicators, City Benchmarking and Real-Time Dashboards," Regional Studies, Regional Science 2: 1 (2015) 6-28.

R. Kitchin, S. Maalsen, and G. McArdle, "The Praxis and Politics of Building Urban Dashboards," Geoforum 77: 1 (2016) 93-101.

R. Kitchin and G. McArdle, "Urban Data and City Dashboards: Six Key Issues," in R. Kitchin, G. McArdle, and T. Lauriault, eds, Data and the City (London: Routledge, 2017) 111-126.

S. Kubler, J. Robert, Y. Le Traon, J. Umbrich, and S. Neumaier, "Open Data Portal Quality Comparison Using AHP," paper presented at the 17th International Digital Government Research Conference on Digital Government Research, Shanghai, June 8-10, 2016).

H. Kuusela and P. Paul, "A Comparison of Concurrent and Retrospective Verbal Protocol Analysis," American Journal of Psychology 113: 3 (2000) 387-404.

T. Lauriault, and M. Francoli, "Openness, Transparency, Participation," in R. Kitchin, G. McArdle, and M. Wilson, eds, Understanding Spatial Media (London: Sage, 2017) 188-203.

C. Lewis, "Thinking-Aloud in User Interface Design: A Method Promoting Cognitive Ergonomics," Ergonomics 33: 4 (1990) 501-507.

M. Lněnička and R. Máchová, “Open (Big) Data and The Importance of Data Catalogs and Portals for the Public Sector," paper presented at Global Virtual Conference: The Third International Global Virtual Conference, Zilina, April 1, 2015.

R. P. Lourenço, "An Analysis of Open Government Portals: A Perspective of Transparency for Accountability," Government Information Quarterly 32: 3 (2015) 323-332.

R. Máchová and M. Lněnička, "Evaluating the Quality of Open Data Portals on the National Level," Journal of Theoretical and Applied Electronic Commerce Research 12: 1 (2017) 21-41.

N. Mack, Qualitative Research Methods: A Data Collector's Field Guide (Research Triangle Park, NC: Family Health International, 2005).

K. Malterud, V. D. Siersma, and A. D. Guassora, "Sample Size in Qualitative Interview Studies: Guided by Information Power," Qualitative Health Research 26: 13 (2016) 1753-1760.

T. Matthews, T. Judge, and S. Whittaker, "How Do Designers and User Experience Professionals Actually Perceive and Use Personas?" paper presented at the SIGCHI Conference on Human Factors in Computing Systems, Austin TX, May 5-10, 2012.

G. McArdle and R. Kitchin, "Improving the Veracity of Open and Real-Time Urban Data," Built Environment 42: 3 (2016a) 446-462.

G. McArdle and R. Kitchin, "The Dublin Dashboard: Design and Development of a Real-Time Analytical Urban Dashboard," ISPRS Annals of the Photogrammetry, Remote Sensing and Spatial Information Sciences 3: 4 (2016b) 19-25. 
S. Mulder and Z. Yaar, The User Is Always Right: A Practical Guide to Creating and Using Personas for The Web (San Francisco: New Riders, 2006).

NIRSA, "Dublin Dashboard" (2014) <www.dublindashboard.ie/pages/index> Accessed October 11, 2018.

J. Nielsen, “The Matters That Really Matter for Hypertext Usability," paper presented at the ACM Hypertext 89 Conference, Pittsburgh PA, November 5-8, 1989.

J. Nielsen, Usability Engineering (Amsterdam: Elsevier, 1994).

J. Pruitt and J. Grudin, "Personas: Practice and Theory," paper presented at the ACM Designing for User Experiences, San Francisco, June 6-7, 2003.

M. B. Rivera, E. Eriksson, and J. Wangel, "ICT Practices in Smart Sustainable Cities," paper in the EnviroInfo and ICT for Sustainability Conference, Copenhagen, September 7-9, 2015.

L. Rosenfeld, and P. Morville, Information Architecture for The World Wide Web (Newton: O'Reilly Media, 2002).

L. Ross, D. Greene, and P. House, “The 'False Consensus Effect': An Egocentric Bias in Social Perception and Attribution Processes," Journal of Experimental Social Psychology 13: 3 (1977) 279-301.

Y. Shin and D. Shin, "Community Informatics and the New Urbanism: Incorporating Information and Communication Technologies into Planning Integrated Communities," Journal of Urban Technology 19: 1 (2012) 23-42.

B. Shneiderman, Designing the User Interface: Strategies for Effective Human-Computer Interaction (Delhi: Pearson Education India, 2010).

Smart London Board, Smart London Plan: Using the Creative Power of New Technologies to Serve London And Improve Londoners' Lives (London: Greater London Authority, 2013).

State of Hawaii, "Open Performance Hawaii" (2014) <https://dashboard.hawaii.gov/> Accessed October 11, 2018.

K. Unsworth, A. Forte, and R. Dilworth, "Urban Informatics: The Role of Citizen Participation in Policy Making," Journal of Urban Technology 21: 4 (2014) 1-5.

G. W. Young and R. Kitchin, "Creating Design Guidelines for Building City Dashboards from a Users' Perspectives," International Journal of Human-Computer Studies, 140 (2020) 102429.

S. Zhu, H. H. Yang, and L. Feng. "Visualizing and Understanding the Digital Divide," paper presented at the International Conference on Hybrid Learning and Continuing Education, Wuhan, July 27-29, 2015.

A. Zuiderwijk, M. Janssen, and C. Davis, "Innovation with Open Data: Essential Elements of Open Data Ecosystems," Information Polity 19 (2014) 17-33. 